\title{
Combination therapy targeting Raf-1 and MEK causes apoptosis of HCT116 colon cancer cells
}

\author{
ROMESH R. SUBRAMANIAN and AKIO YAMAKAWA \\ Department of Cancer Biology, Dana-Farber Cancer Institute and Department of Pathology, \\ Harvard Medical School, Boston, MA 02115, USA
}

Received June 3, 2012; Accepted July 6, 2012

DOI: 10.3892/ijo.2012.1602

\begin{abstract}
Members of the Ras protooncogene family are mutated in approximately $75 \%$ of colon cancers. The Raf kinases (Raf-1, b-Raf and a-Raf) directly interact with Ras and serve as mediators of mitogenic signals. Expression of the constitutively active alleles of Raf or Ras gene families results in oncogenesis in a number of model systems. Previous studies emphasized the importance of Raf- 1 and b-Raf in preventing apoptosis in addition to their roles in cell growth. In the present study, we examined whether inhibition of the Raf- 1 or b-Raf kinase decreases cell growth and increases apoptosis in colon cancer cells. c-Raf and b-Raf were depleted in colon cancer cell lines, such as HCT116, HT29 and Colo205, containing Ras or b-Raf mutations by RNA interference (RNAi). The results showed that colon cancer cells with activating Ras mutations undergo apoptosis following Raf-1 inhibition, as determined by cell cycle analysis and the release of cytochrome $c$. Moreover, in b-Raf mutant colon cancers, the inhibition of b-Raf as compared to Raf-1 is crucial for cancer cell death. There is increasing evidence for both MEK-independent Raf signaling and Raf-independent MEK signaling. Thus, we investigated whether targeting multiple points of the mitogen-activated protein kinase (MAPK) pathway with a MEK inhibitor and Raf RNAi increases cancer cell death. The results showed that combination therapy, inhibiting Raf and MEK kinases simultaneously, increased apoptosis in colon cancer cells. Taken together, our data demonstrate that combination therapy targeting the MAPK pathway at two distinct points, Raf kinase and MEK, has greater efficacy in increasing cancer cell death and is likely to improve therapeutic outcomes for patients.
\end{abstract}

Correspondence to: Dr Romesh R. Subramanian, Department of Cancer Biology, Dana-Farber Cancer Institute and Department of Pathology, Harvard Medical School, Boston, MA 02115, USA

E-mail: romesh.r.subramanian@gmail.com

Key words: Raf, MEK, apoptosis, cancer, RNA interference

\section{Introduction}

Multicellular organisms stringently control the activities of their cells and tissues by a complex network of signaling pathways. This network guarantees that cells grow, proliferate and divide in a systematic manner within the organism. Conditions leading to the deregulation of these signaling pathways often result in cancer. The Ras-Raf-MAPK signaling cascade is crucial in maintaining cell homeostasis, as it plays a diverse role in various biological processes (1). The overexpression or constitutive activation of any one of a large number of receptor tyrosine kinases (RTK) leads to Ras activation. The Ras proteins are products of one of the earliest identified oncogenes, and $\sim 30 \%$ of human epithelial tumors frequently possess activating mutations of a ras gene $(\mathrm{H}-, \mathrm{N}-$ or K-ras) $(2,3)$. Activation of Ras results in the stimulation of downstream signaling cascades of which the Raf-MEK-ERK cascade is the best characterized candidate. Ras has proven to be problematic for intervention by pharmacological agents (4). Consequently, Raf and MEK are regarded as key protein kinase candidates for anticancer drug design. Ras interacts with and activates the Raf family of kinases, which in turn phosphorylate and activate the mitogen-activated protein kinase kinase 1/2 (MEK1/2). MEK ultimately phosphorylates and activates intracellular serine/threonine kinases termed mitogen-activated protein kinases or extracellular signalregulated kinases (MAPK/ERK). The MAPK cascade is an important regulator of cell proliferation, differentiation and apoptosis (5). Thus, blocking the MAPK cascade may provide the means to increase cancer cell death.

Raf kinase is directly downstream of Ras in the MAPK cascade. The Raf kinase family comprises a-Raf, b-Raf and Raf-1, which are thought to have both overlapping and unique regulatory functions (6). Active mutants of Raf- 1 and b-Raf possess transforming activity in vitro and have also been identified in a number of human tumors $(7,8)$. Activating mutations in Ras or RTKs also result in activation of the Raf-1 kinase, thus conferring a growth advantage on the cancer cell. Evidence from mouse knockouts has demonstrated a previously unknown pro-survival role for Raf-1 and b-Raf (9-12). These kinases are essential in the prevention of apoptosis during mouse development. Thus, inhibition of Raf- 1 or b-Raf kinases may sensitize cancer cells to apoptotic stimuli. The pharmacological inhibition of Raf kinases has been extensively 
explored with limited success, and results of anti-c-Raf RNA aptamers and peptides that block Ras-Raf interaction have shown some promise.

To evaluate Raf kinases as targets to block growth or enhance cancer cell death, the expression of Raf-1 and b-Raf was silenced by RNA interference (RNAi) and the effects of this depletion on growth and apoptosis were determined. The effects of MEK inhibition were evaluated in assays used in this study. The results showed that silencing Raf-1 or b-Raf or MEK alone causes a modest increase in cancer cell death. However, a combination treatment that depletes both Raf-1 and MEK provides a significant increase in colon cancer cell death. These data suggest that tumors with activated ras or a hyperactive MAPK cascade (perhaps via constitutively active Ras or b-Raf mutants) may be sensitive to a multi-pronged attack on the MAPK cascade with Raf and MEK inhibitors.

\section{Materials and methods}

Cell culture. Cos-7 cells and cancer cell lines HCT116, HT29, Colo205, ME180 and MCF-7 were purchased from ATCC and propagated in Dulbecco's modified Eagle's medium (DMEM, Invitrogen) supplemented with $10 \%$ fetal bovine serum (FBS) (JRH Biosciences) and 1x penicillin/streptomycin (Invitrogen) at $37^{\circ} \mathrm{C}$ in $10 \% \mathrm{CO}_{2}$.

RNA interference. Cells ( $3 \times 10^{5} /$ well) were plated in 6-well plates (Nalgene). Oligonucleotides encoding the RNA of choice (IDT, Coralville, IA, USA) were subcloned into pSUPER or pSUPERretro (Oligoengine, Seattle, WA, USA) using the Oligoengine protocol. Cancer cells were grown overnight at $37^{\circ} \mathrm{C}$ in $10 \% \mathrm{CO}_{2}$ and then transfected with $1 \mu \mathrm{g}$ of pSUPER-Raf-1 RNAi (GATCCCCGTGATGCTGTCCACTCGGATTCAAGAGAT CCGAGTGGACAGCATCACTTTTTGGAAA) or pSUPER vector alone or pSUPER -b-Raf wt RNAi (GATCCCCGCTA CAGTGAAATCTCGATTTCAAGAGAATCGAGATTTCA CTGTAGCTTTTTGGAAA) or pSUPER-b-Raf VE RNAi (GATCCCCGCTACAGAGAAATCTCGATTTCAAGAGAA TCGAGATTTCTCTGTAGCTTTTTGGAAA), using Lipofectamine $^{\mathrm{TM}} 2000$ (Invitrogen), according to the manufacturer's instructions. After $72 \mathrm{~h}$, cells were lysed in 1\% NP40 lysis buffer and cytosolic lysates were separated by $10 \%$ SDS-PAGE and immunoblotted to detect Raf-1, b-Raf or actin.

Western blotting. Cytosolic lysates were prepared in $1 \%$ NP40 lysis buffer (150 mM NaCl, $10 \mathrm{mM}$ HEPES pH 7.45, $1 \% \mathrm{NP} 40,5 \mathrm{mM}$ each of $\mathrm{Na}_{4} \mathrm{P}_{2} \mathrm{O}_{7}$ and $\mathrm{NaF}, 2 \mathrm{mM} \mathrm{Na}_{3} \mathrm{VO}_{4}$, $10 \mu \mathrm{g} / \mathrm{ml}$ each of Aprotinin and Leupeptin, $1 \mathrm{mM}$ PMSF) and protein concentration was determined using the Bio-Rad DC $^{\text {TM }}$ protein assay. Protein $(25 \mu \mathrm{g})$ was loaded in each well, resolved by $10 \%$ SDS-PAGE and transferred to nitrocellulose membranes (Schleicher \& Schuell). Western blotting was carried out using the following antibodies: Raf-1 and b-Raf (sc-133, sc-5284; Santa Cruz Biotechnology, Inc.), actin (AC-40; Sigma), phospho-MEK and phospho-ERK (9121 and 9101; Cell Signaling), cytochrome $c$ (65981A; BD-Pharmingen), GST (05-311, Upstate Biotechnology). The blots were developed using enhanced chemiluminescence (ECL, Amersham Biosciences, Inc.) and quantified using Bio-Rad Fluor-Max 700.
Cell cycle and apoptosis. Cancer cells transfected with Raf-1/ b-Raf/luciferase shRNA were treated for $24 \mathrm{~h}$ with $5 \mu \mathrm{M}$ MEK inhibitor (U0126) or vehicle (DMSO). Medium containing floating cells was collected and pooled with trypsinized cells, and total cells were obtained by centrifugation at $1000 \mathrm{rpm}$ at $4^{\circ} \mathrm{C}$. Cell pellets were washed with $1 \mathrm{X}$ PBS, resuspended and fixed overnight in $70 \%$ ethanol at $4{ }^{\circ} \mathrm{C}$. Subsequently, the cells were stained with propidium iodide and analyzed by flow cytometry for cell cycle and apoptosis. Similar experiments were conducted in combination with chemotherapeutic drugs (10 nM paclitaxel, $10 \mu \mathrm{M}$ cisplatin).

Cell growth assay. Cells stably transfected with Raf-1/b-Raf/ luciferase shRNA/empty vector were selected and seeded at $5 \times 10^{2}$ cells/well in a 96-well plate. After $24 \mathrm{~h}$, the MTS reagent (CellTiter, Promega, WI, USA) was added to each well $(333 \mu \mathrm{g} / \mathrm{ml}$ MTS $)$ and cells were incubated at $37^{\circ} \mathrm{C}, 10 \%$ $\mathrm{CO}_{2}, 4 \mathrm{~h}$. Bioreduction of formazan by endogenous esterases in viable cells was colorimetrically quantified using a spectrophotometer at $490 \mathrm{~nm}$. The amount of converted formazan was directly proportional to the number of viable cells in culture.

Soft agar assay. Cells were transfected with $1 \mu \mathrm{g}$ pSUPER or pSUPER-retro plasmid (Raf-1 shRNA, b-Raf shRNA or luciferase shRNA) and $0.1 \mu \mathrm{g}$ of pBabe-puromycin plasmid, and selected with $2 \mu \mathrm{g} / \mathrm{ml}$ puromycin. Clones were selected and expanded for 1 month and analyzed for Raf- 1 or b-Raf protein by western blotting. Cells $\left(2 \times 10^{4}\right)$ in DMEM $/ 10 \%$ FBS were suspended in $1.5 \mathrm{ml}$ of $0.3 \%$ agarose and seeded in triplicate in $35-\mathrm{mm}$ plates coated with $1.5 \mathrm{ml}$ of $0.6 \%$ agarose/ DMEM $/ 10 \%$ FBS. Cells were grown at $37^{\circ} \mathrm{C}$ in $10 \% \mathrm{CO}_{2}$ and the number of colonies formed was scored after 2 weeks.

\section{Results}

Raf-1 and b-Raf silencing by RNAi. To determine whether therapeutic intervention in the MAPK cascade at Raf is feasible and potentially effective in killing cancer cells, we initially silenced Raf-1 expression using RNAi. As shown in Fig. 1A, Raf-1 was effectively silenced in epithelial cancer cells using three different target sites for shRNAs directed against c-Raf. A control shRNA vector targeting Hsp70 or luciferase did not deplete Raf-1, revealing the specificity of Raf-1 silencing. The expression of shRNA targeting b-Raf wt did not alter Raf-1 protein levels, and vice versa (Fig. 2B and Fig. 1B). Subsequent experiments were carried out using the shRNA vector, which targets site 1 (Raf-1 \#1 in Fig. 1A). Raf-1 silencing resulted in a modest decrease in ERK1/2 phosphorylation but did not change the total ERK expression (Fig. 1A). Additionally, shRNA constructs targeting b-Raf wt (Fig. 1B) and b-Raf VE (Fig. 1C) selectively inhibited the specific protein expression.

Raf-1 depletion in HCT116 does not alter proliferation but increases apoptosis. Raf-1 is a key mediator of the MAPK cascade in cells and its activation ultimately facilitates cell growth, differentiation and proliferation. Moroever, it is reported to protect cells from apoptosis. Based on these data, we expected that silencing of Raf-1 would decrease cancer cell growth and/or increase their apoptotic index. To test this model, we utilized HCT116 colon cancer cells that either 
A
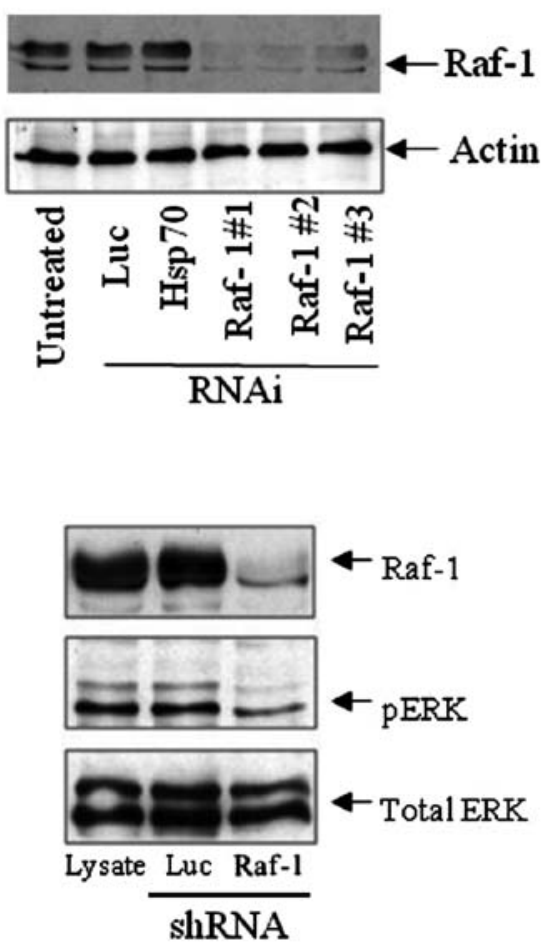

$\mathrm{B}$

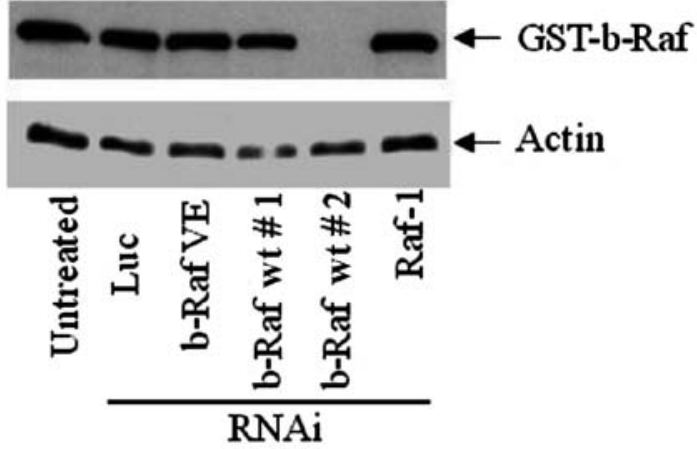

C
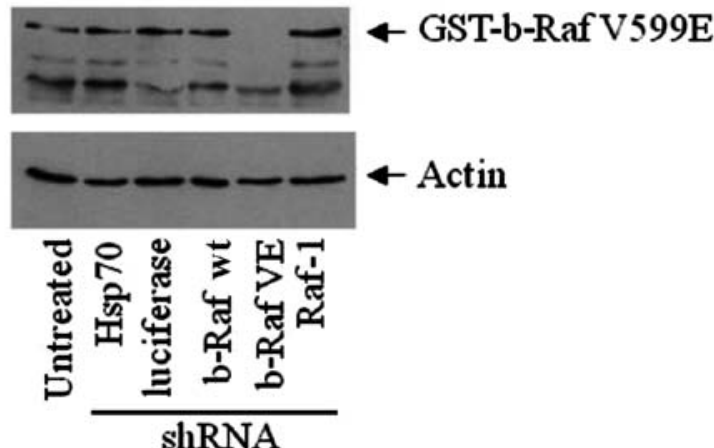

Figure 1. Characterization of oligo- and vector-based RNAi of human c-Raf and b-Raf. (A) Efficacy of RNAi oligomers and shRNA vectors in inhibiting Raf expression. The top panel shows that subconfluent Cos-7 cells were either transfected with shRNA directed against Hsp70 or luciferase or with the series of shRNAs directed against endogenous c-Raf using Lipofectamine ${ }^{\mathrm{TM}} 2000$ (Invitrogen). After a 72-h transfection, cytosolic lysates were prepared and tested for Raf-1 expression by western blotting $(n=3)$. Actin was used as a loading control. The bottom panel shows that Cos-7 cells were transfected with pSUPER-retro shRNA vector targeting c-Raf (Raf-1 \#1) using FuGene6, and the expression of Raf-1 as well as phospho- and total-ERK was determined by western blotting. (B and C) Cos-7 cells were co-transfected with pDEST27-GST-bRaf wt, or -bRaf V599E. The suppression of specific b-Raf expression was determined by western blotting for GST.

stably expressed pSUPER-retro targeting luciferase or Raf-1 or b-Raf wt under puromycin selection, and, using an MTS assay, examined whether these cell lines exhibited any differences in growth rate and viability. The growth rate of HCT116 (K-Ras G13D) did not decrease significantly upon the expression of shRNA directed against Raf-1, b-Raf or luciferase (Fig. 2A). Cell cycle analysis revealed that the Raf-1-deprived HCT116 cells had higher levels of apoptosis, observed as an increased sub-G0 population (Fig. 2B). Cells with decreased b-Raf and control cells expressing an shRNA to luciferase, exhibited similar levels of apoptosis as the untreated cells.

Increase in cell apoptosis upon b-Raf and Raf-1 silencing. Using mouse knockout models, two Raf genes ( $b$ - and $c$-Raf) were previously shown to play a key role in preventing cell apoptosis during development (9-11). However, these mouse knockouts were embryonic lethal. To determine whether these Raf kinases protect differentiated cells from apoptosis, we silenced either Raf- 1 or b-Raf expression using RNAi in HCT116, HT29 and Colo205 cells and determined cell apoptosis by DNA content-based flow cytometry. These cells express either mutant K-Ras G13D (HCT116) or mutant b-Raf V599E (HT29 and Colo205). DNA content-based flow cytometry analysis revealed that HCT116 (K-Ras G13D/Raf-1 wt/ b-Raf wt) had increased apoptosis when Raf-1 was silenced, but b-Raf wt or b-Raf VE silencing did not increase apoptosis above control levels (Fig. 3A). Cisplatin treatment was used as a positive control for cell apoptosis, while treatment with pSUPER-luciferase was the negative control. HT29 cells (Ras wt/Raf-1 wt/b-Raf wt: VE), which are heterozygous for the activating b-Raf mutation V599E, showed increased apoptosis when b-Raf VE was silenced as compared to when Raf-1 or b-Raf wt were silenced (Fig. 3B). However, Colo205 (Ras wt/ Raf-1 wt/b-Raf VE: VE), which are homozygous for b-Raf V599E, were unresponsive to the depletion of Raf-1 or b-Raf wt. Notably, silencing of b-Raf V599E in Colo205 cells caused a marked increase in apoptosis as compared to the control (Fig. 3C). Our results demonstrate that inhibition of the specific Raf kinases in cancer cells dependent on oncogenes such as ras or $b$-Raf, are likely to increase the apoptotic index and contribute to cancer cell death.

Raf-1 and b-Raf enhance anchorage-independent growth in soft agar. To test the potential of Raf kinase inhibition in cancer therapy, we carried out soft agar colony formation assays. Raf-1 or b-Raf was silenced by RNAi in HCT116 cells and stable cell lines were generated as described earlier. These stable cell lines were grown in soft agar to detect their ability to form colonies in an anchorage-independent manner. Cell lines with luciferase-targeted shRNA formed many small colonies in soft agar within two weeks (Fig. 4, upper panel). However, silencing of Raf-1 in HCT116 cells caused 

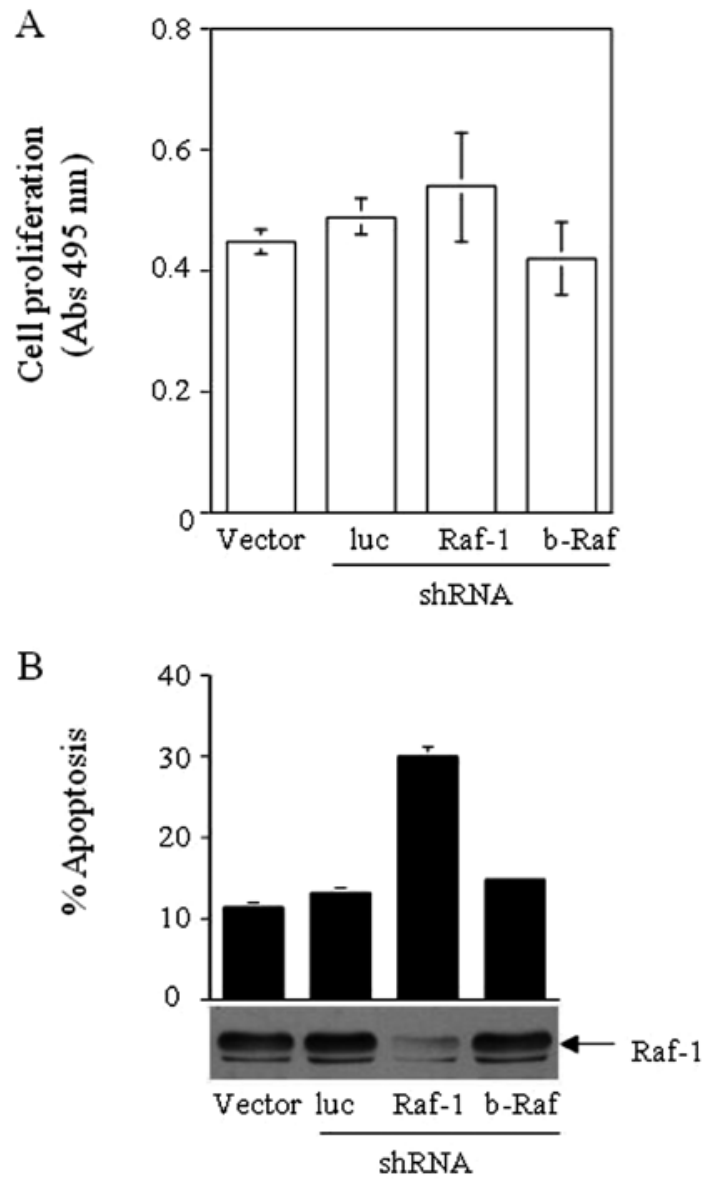

Figure 2. Effect of Raf-1 or b-Raf depletion on cell proliferation and apoptosis in HCT116 cells. HCT116 cells were stably transfected with shRNA vectors targeting luciferase, c-Raf or b-Raf wt. (A) Cell growth/viability was determined using the MTS assay. (B) Apoptosis was evaluated by flow cytometry to identify small fragmented DNA and Raf-1 expression was visualized by western blotting.

a notable decrease in soft agar colony formation, confirming the importance of Raf-1 kinase activity for anchorage-independent cell growth (Fig. 4, middle panel). However, b-Raf silencing in HCT116 cells was not as effective at inhibiting soft agar colony formation, although it modestly decreased soft-agar growth (Fig. 4, lower panel). Previous experiments proved that silencing Raf-1 or b-Raf did not significantly alter the growth rate of these cells (Fig. 2A). Conversely, analysis of cell apoptosis by flow cytometry demonstrated that Raf-1 silencing resulted in increased apoptosis of the cells used in the soft agar assay (Fig. 2B). These results may explain the decreased rate of colony formation in soft agar upon Raf-1 silencing.

Combination of Raf-1 depletion and MEK inhibition increases cancer cell death. Previous studies have shown that Raf-1 activation enhances cellular resistance to chemotherapeutic drug treatments $(13,14)$. We tested whether Raf- 1 was important for colon cancer cell sensitivity to the chemotherapeutic agent, paclitaxel. HCT116 colon cancer cells, with a stable expression of either pSUPER-luciferase (Luc) or pSUPER-Hsp70 shRNA as negative control or pSUPERRaf-1 shRNA, were treated with $10 \mathrm{nM}$ paclitaxel or DMSO for $16 \mathrm{~h}$. After $16 \mathrm{~h}$ of paclitaxel treatment, the cells were
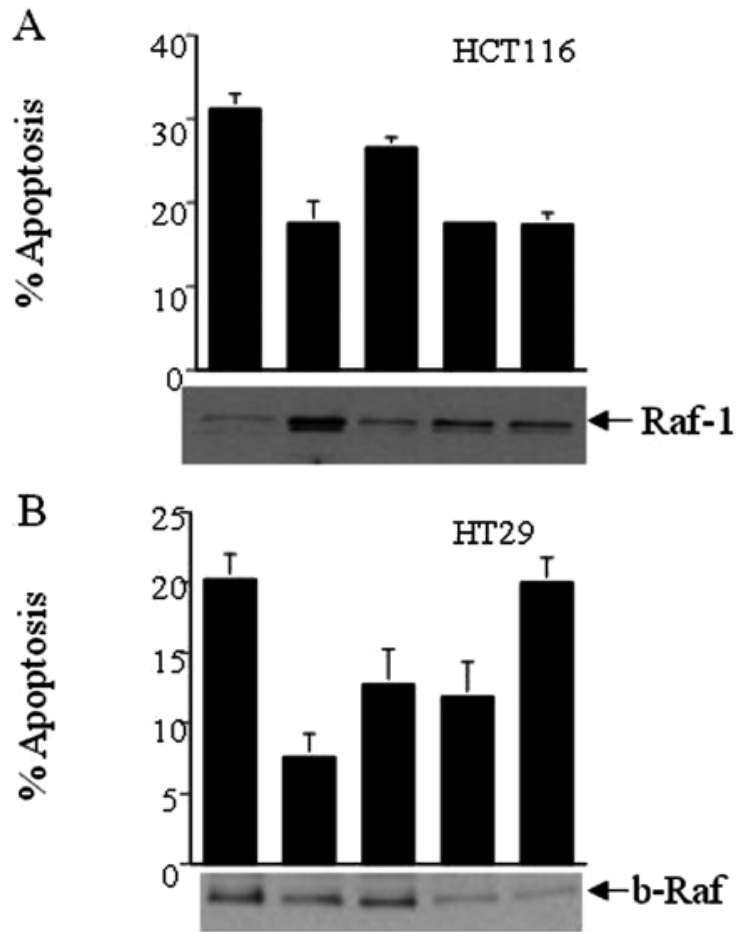

C

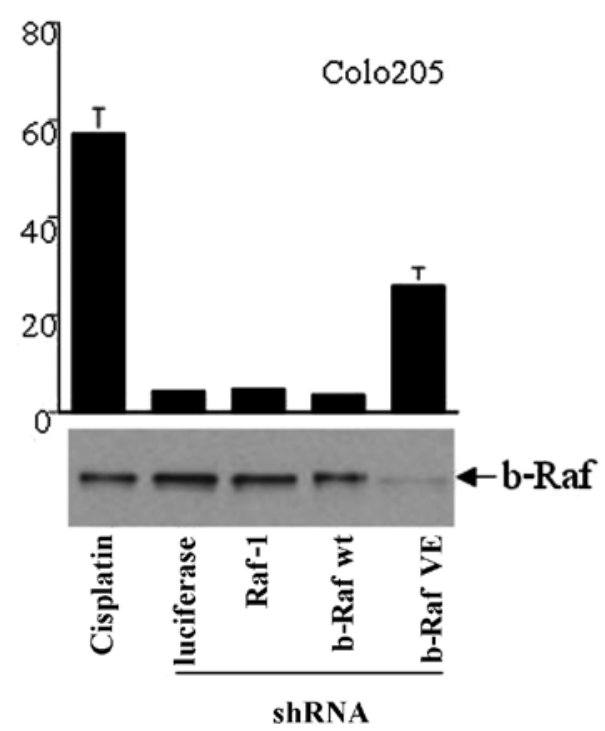

Figure 3. Consequence of Raf-1 and b-Raf depletion on cancer cell death Colon cancer cells HCT116, HT29 and Colo205 underwent stable transfection with pSUPER-retro shRNA vectors directed against c-Raf, b-Raf-wt, b-Raf-V599E or luciferase. Following puromycin selection, cell apoptosis was analyzed by DNA-content based flow cytometry. Cisplatin $(10 \mu \mathrm{M})$ was used as a positive control for the apoptotic assay.

fixed, DNA was stained with propidium iodide and cells were analyzed for apoptosis by flow cytometry (Fig. 5A). Silencing of Raf-1 resulted in increased sensitivity to paclitaxel as evidenced by the increased apoptosis in these cells compared to cells treated with paclitaxel only, and this corresponded to increased cytochrome $c$ release (Fig. 5B).

In previous studies, Raf-1 was found to have a MEK-independent role in signaling. Conversely, MEK/MAPK signaling potentially occurs independent of Raf-1 $(15,16)$. Therefore, we assessed whether a multi-pronged inhibition of the Ras-RafMAPK cascade enhances cancer cell death when compared to Raf or MEK-1 inhibition alone. HCT116 (colon cancer) 

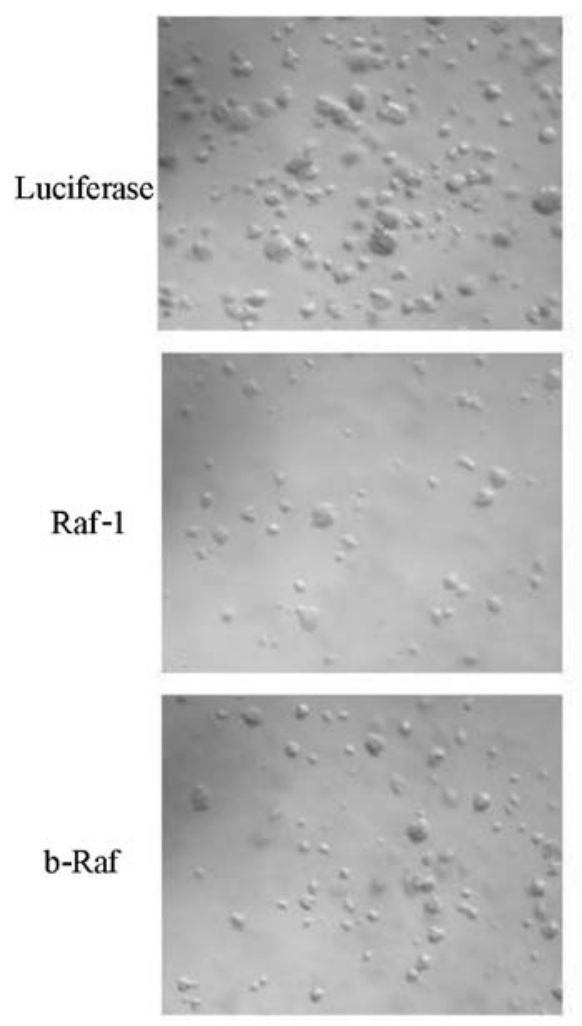

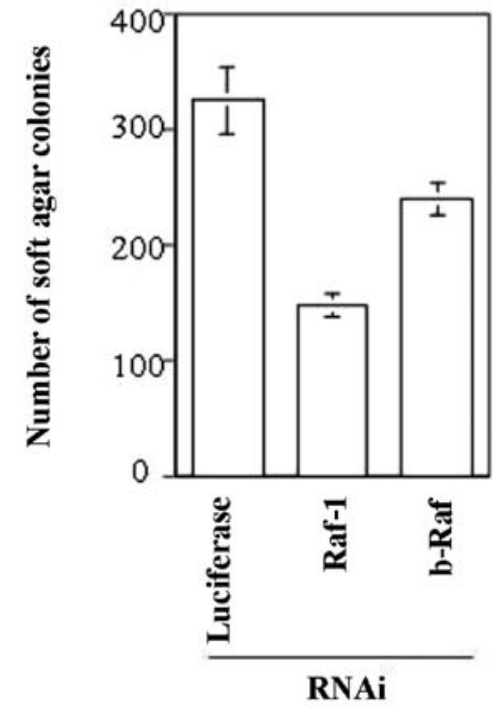

Figure 4. Effect of stable suppression of Raf-1 or b-Raf wt on anchorage-independent growth. HCT116 cells were stably transfected with pSUPER-retro shRNA vector targeting c-Raf, b-Raf or luciferase. After puromycin selection, the cells were suspended in agarose and the number of colonies formed after 14 days was observed (left panel, magnification, $x 20$ ) and scored if $>0.2 \mathrm{~mm}$ in diameter (right panel). Results are representative of two independent experiments carried out in triplicate.

and ME-180 (cervical cancer) cells were employed to test this hypothesis by silencing Raf-1 with shRNA and treating the cells with a MEK inhibitor (5 $\mu \mathrm{M}$ U0126). Cells treated with Raf-1 shRNA and vehicle (DMSO) had a moderate 2 -fold increase in apoptosis compared to the vehicle and luciferase shRNA or Hsp70 shRNA-treated cells (Fig. 6A and B). MEK-1 inhibition results in greater apoptosis than Raf-1 silencing alone in both HCT116 and ME-180 cells. Notably, the co-treatment of cancer cells with Raf-1 shRNA and MEK inhibitor resulted in a marked increase in cell apoptosis in HCT116 (Fig. 6A) and ME-180 (Fig. 6B) cells. Thus, cancer cells may possess a MEK-independent signaling function for Raf-1. Such a phenomenon has the potential to be exploited for therapeutic purposes by efficiently blocking the entire MAPK cascade simultaneously with inhibitors for Raf kinase as well as MEK kinase.

\section{Discussion}

Cancer is known to be a multistage process (17) caused by the progressive accumulation of activating mutations in dominant growth-enhancing genes/oncogenes and inactivating mutations in growth-suppressing/tumor suppressor genes (18). To maintain cell homeostasis, cancer cells may be addicted to the activity of specific oncogenes as opposed to normal cells. This oncogene addiction is beneficial to the cancer cell by way of conferring certain growth advantages upon it. However, inhibition of the oncogene function may lead to collapse of cell homeostasis and cancer cell death (19-22). Approximately $30 \%$ of solid tumors carry activating Ras mutations, and epithelial tumors have been shown to possess a hyperactive Ras-RafMAPK pathway (23). Recently, activating mutations in b-Raf (particularly V599E) have been found in over $60 \%$ of melanomas and in approximately $15 \%$ of colon cancers (8). These data led to the hypothesis that inhibition at any point of the Ras-Raf-MAPK pathway in cancer cells is likely to diminish growth-promoting signals, and thus tilt the cellular balance in favor of death-promoting pathways. To test this hypothesis, we targeted the Ras-Raf-MAPK pathway in cancer cells at two separate points by silencing the Raf protein expression using RNAi together with the pharmacological inhibition of MEK.

In this study, we have demonstrated that Raf kinases are effectively depleted or silenced in cancer cells by RNAi. The targeting of Raf-1 and b-Raf by RNA interference was highly specific as evidenced by the lack of Raf kinase depletion by RNAi of Hsp70 or luciferase (Fig. 1). In addition, RNAi of Raf-1 reduces but does not eliminate downstream effector signaling such as ERK phosphorylation (Fig. 1A), indicating that Raf-1 may not be the only molecule signaling via ERK. ERK is important in modulating cell growth and proliferation as well as resistance to apoptosis $(24,25)$, and ERK is hyperactive in colon cancers possessing activated K-Ras (26). Raf-1 signaling via ERK is also associated with increased mdrl or drug efflux pump expression, which is able to increase cellular resistance to chemotherapeutic drug treatment by higher drug efflux from the cell (27). Of note, ERK activation has been shown to result in a positive feedback to Raf-1 thereby amplifying the Raf-MAPK cascade and promoting cell survival and 


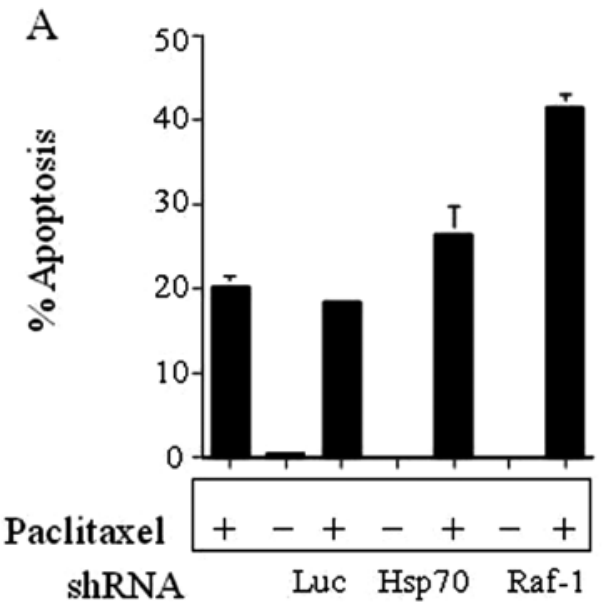

B

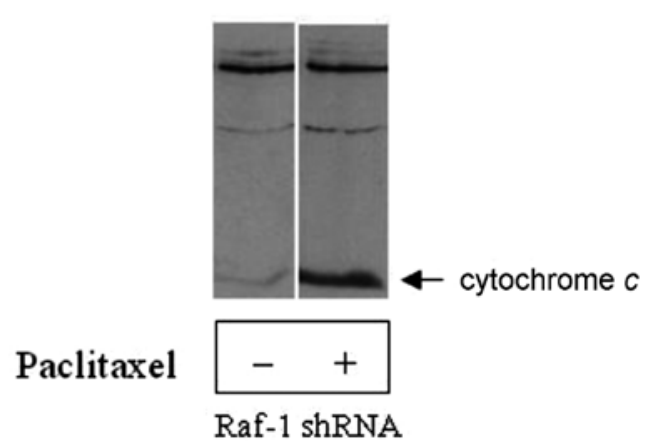

Figure 5. Consequence of Raf-1 depletion on HCT116 cellular sensitivity to paclitaxel. (A) HCT116 cells were stably transfected with shRNA targeting Hsp70, luciferase or c-Raf. After a 54-h transfection and puromycin selection, the cells were treated with $10 \mathrm{nM}$ paclitaxel or DMSO (vehicle) for $16 \mathrm{~h}$. Cell apoptosis was quantified by flow cytometry and the sub-G0 fraction (fragmented DNA) is shown on the Y-axis. (B) Cytosolic lysates from the above-mentioned cells were analyzed for the presence of cytochrome $c$, an apoptosis indicator, by western blotting $(\mathrm{n}=3)$.

growth (28). Thus, even a modest reduction in ERK activation is likely to result in decreased cell growth, survival and increased susceptibility of the cancer cells to apoptotic stimuli.

In our experiments, silencing of Raf-1 reduced ERK phosphorylation, but did not decrease cancer cell growth. MTS assays demonstrated that cancer cells such as HCT116 or MCF-7, with or without a lower expression of Raf-1/b-Raf, had similar culture growth rates. These data suggest that Raf kinases are not crucial for cell growth. However, it is possible that the low level of ERK activation still present is sufficient for cell growth. Cyclin B-Cdc2, a master mitosis regulator, has been reported to activate MEK1 during mitosis without subsequent ERK activation (29). Thus, it is possible that Raf kinases have a reduced role during cell division and growth.

Recent mouse knockout models for Raf kinases have shown that Raf-1 and b-Raf play critical roles during development $(9,10)$, primarily in preventing apoptosis. ERK activation is also involved in the phosphorylation and proteasomal degradation of the pro-apoptotic BH3-only protein Bim (30), thus preventing cell death. Our data support these findings since the depletion of Raf-1 or b-Raf by RNAi increased cellular apoptosis. Notably, we found that cancer cells with certain potential oncogene addictions, such as activated K-Ras, were more susceptible to apoptosis via the inhibition of Raf-1
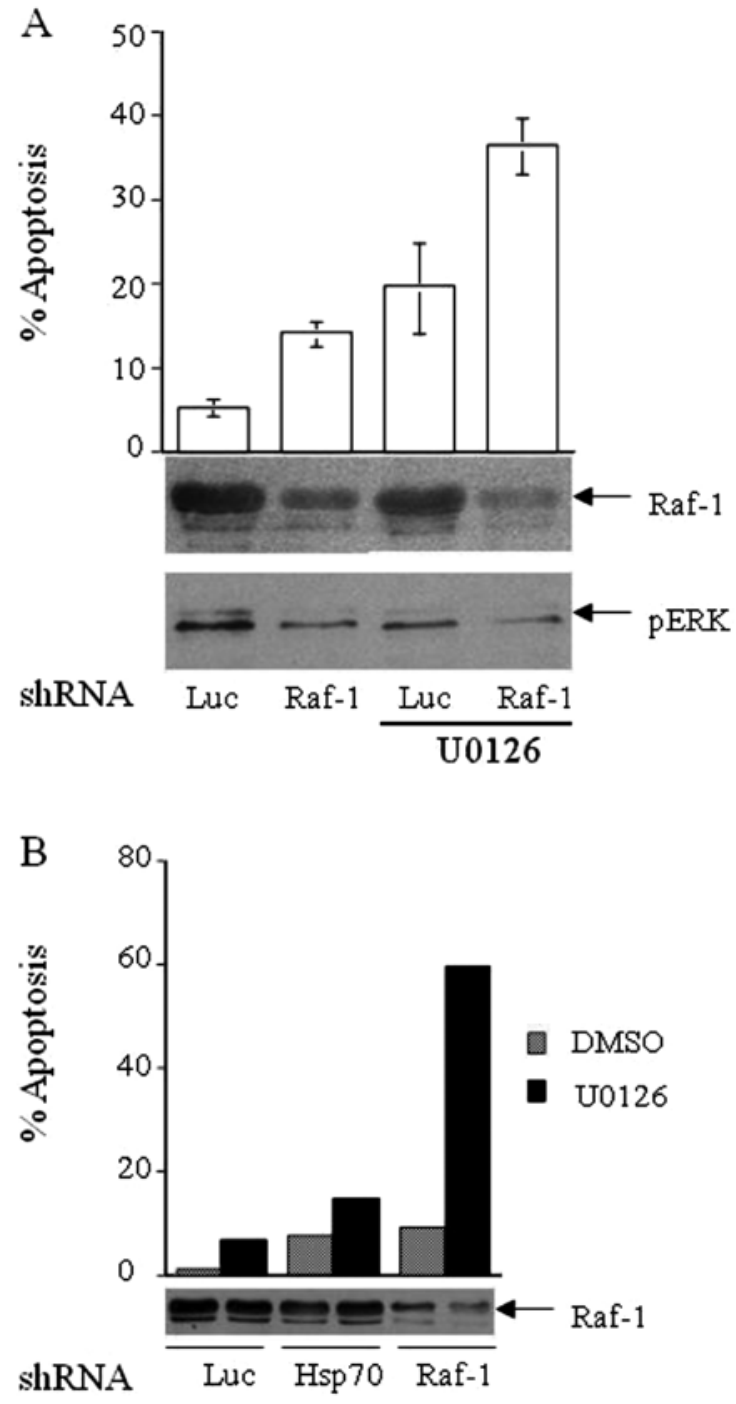

Figure 6. Results of the combination treatment using MEK inhibition and Raf-1 depletion on HCT116 and ME-180 cells. Cancer cells stably transfected with shRNA targeting c-Raf, luciferase or Hsp70 were treated with either $5 \mu \mathrm{M}$ U0126 or DMSO. Cell apoptosis was measured by flow cytometry. Expression of Raf-1 and phospho-ERK were determined by western blotting in HCT116 (panel A) and ME-180 (panel B) cells.

kinase as compared to b-Raf. Similarly, cancer cells with the activating b-Raf mutant (V599E) were better targeted by b-Raf RNAi compared to the RNAi of Raf-1. HCT116 cells that possess activated K-Ras (G13D) exhibited greater apoptosis when Raf-1 was depleted, suggesting that Raf-1 is the primary MAPKKK for Ras-mediated signaling in these cells. However, HCT116 cells were refractory to b-Raf silencing, confirming previous studies that cancers with an activating mutation in ras seldom possess an accompanying mutation in $b$-Raf (12), since they probably preferentially signal via alternate molecules such as Raf-1. Colon cancer cells which were either heterozygous (HT29) or homozygous (Colo205) for the activating b-Raf mutation (V599E) exhibited greater sensitivity to abrogation of signaling from the b-Raf mutant. Similarly, b-Raf V599E-dependent melanoma cells (A375) were sensitive to the RNAi-mediated silencing of b-Raf V599E (data not shown). These data suggest that the primary role of Raf kinases is to protect cells from apoptosis. Results 
obtained from the soft agar assays supported this hypothesis. This assay is a stringent test of the ability of cancer cells to grow in an anchorage-independent manner. A strong correlation exists between the ability of cells to grow in soft agar and their ability to form tumors in vivo, although the correlation is not absolute. Comparison of soft agar growth in the presence and absence of Raf-1 or b-Raf demonstrated noteworthy differences. Specifically, HCT116 and MCF-7 cells depleted of Raf-1 showed greater apoptosis by flow cytometry and much fewer colonies in soft agar compared to control or b-Raf depletion, suggesting that these cells are primarily dependent on Raf-1 for MAPK signaling and protection from apoptosis. Additionally, the molecular targeting of cancers based on the activated oncogene is a potentially viable therapeutic strategy that could contribute to a greater understanding of personalized medicine.

Since Raf participates in preventing cell death, it follows that depletion/silencing of Raf may sensitize cancer cells to concomitant chemotherapeutic drug treatment. Results of this study have shown that Raf-1 silencing in HCT116 or ME-180 cells confers markedly greater susceptibility to paclitaxel treatment. Previous studies have shown that cervical cancer cells with inherently low levels of Raf-1 exhibit paclitaxel sensitivity (13). In this study, cancer cell lines with high Raf-1 expression levels were used and modest levels of apoptosis with paclitaxel treatment were observed. However, silencing of Raf-1 expression by RNAi increased paclitaxel-mediated cytotoxicity. A possible explanation is that Raf depletion may decrease mdr1 expression and efflux of paclitaxel. This reiterates the need for effective Raf kinase inhibitors, so that combination treatment regimens may be employed in the treatment of such cancers.

The dual specificity kinase MEK plays a key role in integrating extracellular signals into the MAPK cascade. Activating ras mutations in cancers result in hyperactive MEK, and MEK activity could be targeted to prevent growth of these cancer cells. Kinase inhibitors targeting MEK have been shown to suppress colon tumor growth in vivo (31), however results of clinical trials have been disappointing. MEK inhibitors have been shown to be primarily cytostatic and not cytotoxic to cancer cells. Recent evidence has, however, raised questions pertaining to the exclusivity of the Raf/MEK/ERK pathway. Mouse knockouts of Raf-1 are embryonic lethal, however, the knockin of an allele of $c$-Raf, mutant at Y340/341F, rendered the knockouts viable. These mice lacked detectable MEK activity but exhibited normal ERK activity and cell proliferation (15). Raf has also been involved in direct translocation to the mitochondria and subsequent phosphorylation of $\mathrm{Bad}$, thus enhancing 14-3-3 binding and blocking of Bad-mediated apoptotic activity (32-35). ASK1, a stress-induced pro-apoptotic protein, is another noteworthy binding partner of Raf-1. Notably ASK1 is inhibited by either wild-type or kinase dead Raf-1 (36). Raf-1 has also been reported to induce NF- $\kappa B$ via MEKK1 bypassing MEK (37). Activating mutations of MEK were shown to be capable of transforming NIH3T3 cells in the absence of ERK activation (38). Therefore, mounting evidence indicates a possible MEK-independent role for Raf-1, which suggests that blockade of the MAPK pathway at either Raf-1 or MEK may not be sufficient to block cell survival and induce apoptosis. Thus, we tested whether combination therapy targeting both Raf-1 and MEK was able to increase apoptosis in cancer cells. Our findings have shown that simultaneous Raf-1 RNAi and pharmacological MEK inhibition results in greater cell death in HCT116 colon cancer cells and ME-180 cervical cancer cells than either treatment alone. These are some of the first results demonstrating that dual targeting of a single signaling pathway at two points or nodes may be more efficacious than any single inhibitor alone. Thus, Raf-1 and b-Raf kinases potentially serve as focal points for the inhibition of cancer cell survival.

In summary, our study suggests that multi-pronged targeting of the MAPK pathway in cancer cells with activating mutations in Ras or b-Raf is likely to increase the apoptosis of these cancer cells, and it stresses the need for better pharmacological inhibitors of Raf kinases.

\section{Acknowledgements}

We thank Dr Jean Zhao, Dr Kai Xia and Dr Radha Narsimhan and members of the Roberts Lab for helpful and enlightening discussions.

\section{References}

1. Downward J: Ras signalling and apoptosis. Curr Opin Genet Dev 8: 49-54, 1998.

2. Barbacid M: Ras genes. Annu Rev Biochem 56: 779-827, 1987.

3. Kiaris H and Spandidos D: Mutations of ras genes in human tumours (Review). Int J Oncol 7: 413-421, 1995.

4. Downward J: Targeting RAS signalling pathways in cancer therapy. Nat Rev Cancer 3: 11-22, 2003.

5. Fang JY and Richardson BC: The MAPK signalling pathways and colorectal cancer. Lancet Oncol 6: 322-327, 2005.

6. Chong H, Vikis HG and Guan K-L: Mechanisms of regulating the Raf kinase family. Cell Signal 15: 463-469, 2003.

7. Storm SM, Brennscheidt U, Sithanandam G and Rapp UR: Raf oncogenes in carcinogenesis. Crit Rev Oncog 2: 1-8, 1990.

8. Davies H, Bignell GR, Cox C, et al: Mutations of the BRAF gene in human cancer. Nature 417: 949-954, 2002.

9. Mikula M, Schreiber M, Husak Z, et al: Embryonic lethality and fetal liver apoptosis in mice lacking the c-raf-1 gene. EMBO J 20: 1952-1962, 2001

10. Wojnowski L, Zimmer AM, Beck TW, et al: Endothelial apoptosis in Braf-deficient mice. Nat Genet 16: 293-297, 1997.

11. Wojnowski L, Stancato LF, Larner AC, Rapp UR and Zimmer A: Overlapping and specific functions of Braf and Craf-1 protooncogenes during mouse embryogenesis. Mech Dev 91: 97-104, 2000.

12. Rajagopalan H, Bardelli A, Lengauer C, Kinzler KW, Vogelstein B and Velculescu VE: Tumorigenesis: RAF/RAS oncogenes and mismatch-repair status. Nature 418: 934, 2002.

13. Britten RA, Perdue S, Opoku J and Craighead P: paclitaxel is preferentially cytotoxic to human cervical tumor cells with low Raf-1 kinase activity: implications for paclitaxel-based chemoradiation regimens. Radiother Oncol 48: 329-334, 1998.

14. Rasouli-Nia A, Liu D, Perdue S and Britten RA: High Raf-1 kinase activity protects human tumor cells against paclitaxelinduced cytotoxicity. Clin Cancer Res 4: 1111-1116, 1998.

15. Huser M, Luckett J, Chiloeches A, et al: MEK kinase activity is not necessary for Raf-1 function. EMBO J 20: 1940-1951, 2001.

16. Murakami MS and Morrison DK: Raf-1 without MEK? Sci STKE, 2001: pe30, 2001.

17. Nowell PC: The clonal evolution of tumor cell populations. Science 194: 23-28, 1976.

18. Kinzler KW and Vogelstein B: Landscaping the cancer terrain. Science 280: 1036-1037, 1998.

19. Jain M, Arvanitis C, Chu K, et al: Sustained loss of a neoplastic phenotype by brief inactivation of MYC. Science 297: 102-104, 2002 .

20. Felsher DW and Bishop JM: Reversible tumorigenesis by MYC in hematopoietic lineages. Mol Cell 4: 199-207, 1999. 
21. Pelengaris S, Littlewood T, Khan M, Elia G and Evan G: Reversible activation of c-Myc in skin: induction of a complex neoplastic phenotype by a single oncogenic lesion. Mol Cell 3: 565-577, 1999.

22. Chin L, Tam A, Pomerantz J, Wong M, et al: Essential role for oncogenic Ras in tumour maintenance. Nature 400: 468-472, 1999.

23. Barbacid M: Ras oncogenes: their role in neoplasia. Eur J Clin Invest 20: 225-235, 1990.

24. Anderson P: Kinase cascades regulating entry into apoptosis Microbiol Mol Biol Rev 61: 33-46, 1997.

25. Rice PL, Goldberg RJ, Ray EC, Driggers LJ and Ahnen DJ Inhibition of extracellular signal-regulated kinase $1 / 2$ phosphorylation and induction of apoptosis by sulindac metabolites. Cancer Res 61: 1541-1547, 2001.

26. Bos JL, Fearon ER, Hamilton SR, et al: Prevalence of ras gene mutations in human colorectal cancers. Nature 327: 293-297, 1987.

27. Zhou G and Kuo MT: NF- $\kappa$ B-mediated induction of mdr1b expression by insulin in rat hepatoma cells. J Biol Chem 272: 15174-15183, 1997.

28. Zimmermann S, Rommel C, Ziogas A, Lovric J, Moelling K and Radziwill G: MEK1 mediates a positive feedback on Raf-1 activity independently of Ras and Src. Oncogene 15: 1503-1511, 1997.

29. Harding A, Giles N, Burgess A, Hancock JF and Gabrielli BG: Mechanism of mitosis-specific activation of MEK1. J Biol Chem 278: 16747-16754, 2003.

30. Ley R, Balmanno K, Hadfield K, Weston C and Cook SJ: Activation of the ERK1/2 signaling pathway promotes phosphorylation and proteasome-dependent degradation of the BH3-only protein, Bim. J Biol Chem 278: 18811-18816, 2003.
31. Sebolt-Leopold JS, Dudley DT, Herrera R, et al: Blockade of the MAP kinase pathway suppresses growth of colon tumors in vivo. Nat Med 5: 810-816, 1999.

32. Majewski M, Nieborowska-Skorska M, Salomoni P, et al: Activation of mitochondrial Raf-1 is involved in the antiapoptotic effects of Akt. Cancer Res 59: 2815-2819, 1999.

33. Fang X, Yu S, Eder A, et al: Regulation of BAD phosphorylation at serine 112 by the Ras-mitogen-activated protein kinase pathway. Oncogene 18: 6635-6640, 1999.

34. Zhong J, Troppmair J and Rapp UR: Independent control of cell survival by Raf-1 and Bcl-2 at the mitochondria. Oncogene 20: 4807-4816, 2001.

35. Subramanian RR, Masters SC, Zhang $\mathrm{H}$ and $\mathrm{Fu} \mathrm{H}$ : Functional conservation of 14-3-3 isoforms in inhibiting bad-induced apoptosis. Exp Cell Res 271: 142-151, 2001.

36. Chen J, Fujii K, Zhang L, Roberts T and Fu H: Raf-1 promotes cell survival by antagonizing apoptosis signal-regulating kinase 1 through a MEK-ERK independent mechanism. Proc Natl Acad Sci USA 98: 7783-7788, 2001.

37. Baumann B, Weber CK, Troppmair J, et al: Raf induces NF-kappaB by membrane shuttle kinase MEKK1, a signaling pathway critical for transformation. Proc Natl Acad Sci USA 97: 4615-4620, 2000.

38. Alessandrini A, Greulich H, Huang W and Erikson RL: Mek1 phosphorylation site mutants activate Raf-1 in NIH 3T3 cells. J Biol Chem 271: 31612-31618, 1996. 